\title{
Continuous essential selections and integral functionals
}

\author{
Ari-Pekka Perkkiö *
}

June 24, 2018

\begin{abstract}
Given a strictly positive measure, we characterize inner semicontinuous solid convex-valued mappings for which continuous functions which are selections almost everywhere are selections. This class contains continuous mappings as well as fully lower semicontinuous closed convex-valued mappings that arise in variational analysis and optimization of integral functionals. The characterization allows for extending existing results on convex conjugates of integral functionals on continuous functions. We also give an application to integral functionals on left continuous functions of bounded variation. Keywords: Set-valued and variational analysis Continuous selections Integral functionals Convex duality
\end{abstract}

\section{Introduction}

Given a set-valued mapping $\Gamma$ from a topological space $\mathbb{T}$ to another $X$ and a strictly positive 1 countably additive Borel measure $\mu$ on $\mathbb{T}$, we say that a function $y: \mathbb{T} \rightarrow X$ is an essential selection of $\Gamma$ if $y_{t} \in \Gamma_{t} \mu$-almost everywhere. In this article we study in which situation continuous essential selections are selections. This is important when deriving formulae for convex conjugates of integral functionals on continuous functions. Such conjugates arise in several areas of variational analysis and optimization including optimal control, plasticity theory and mathematical finance, see [2, 3, 6, 9, 15] and references therein.

A function, which belongs to the domain of an integral functional, is an essential selection of the domain of the integrand. However, without further conditions such function is not necessarily a selection of the domain. The articles [6, 14] study convex conjugates of integral functionals on continuous functions. In [14, Theorem 5] Rockafellar assumes full lower semicontinuity, which implies that continuous essential selections are selections of the closure of the domain, whereas in [6, Example 5] this property was assumed explicitly.

${ }^{*}$ Department of Mathematics and Systems Analysis, Aalto University, P.O. Box 11100, FI-00076 Aalto, Finland, ari-pekka.perkkio@aalto.fi

${ }^{1} \mu$ is strictly positive if $\mu(O)>0$ for every nonempty open $O$. 
We show that, for an inner semicontinuous solid convex-valued mapping, continuous essential selections are selections if and only if the mapping has a property which we call outer regularity in measure. While full lower semicontinuity is a purely topological notion, outer regularity in measure takes into account the underlying measure. When $\mathbb{T} \subset \mathbb{R}^{n}$, we prove that fully lower semicontinuous closed convex-valued mappings are outer regular in measure which allows for a generalization [14, Theorem 5] about convex conjugates of integral functionals on continuous functions. We also give an application to integral functionals on left continuous functions of bounded variation. These results have further applications to problems of Bolza; see [12].

\section{Outer regularity in measure}

We denote by $\mathscr{H}_{t}$ and $\mathscr{H}_{x}$ the neighborhood systems of $t \in \mathbb{T}$ and $x \in X$, respectively. We will denote by $\mathscr{H}_{x}^{o}$ the system of open neighborhoods of $x \in X$. The interior and closure of a set $A$ will be denoted by $\operatorname{int} A$ and $\operatorname{cl} A$.

Let $\Gamma: \mathbb{T} \rightrightarrows X$ be a set-valued mapping. The outer limit and inner limit are, respectively,

$$
\begin{aligned}
& (\limsup \Gamma)_{t}=\bigcap_{B \in \mathscr{H}_{t}} \mathrm{cl}\left(\bigcup_{t^{\prime} \in B} \Gamma_{t^{\prime}}\right), \\
& (\liminf \Gamma)_{t}=\bigcap_{B \in \mathscr{H}_{t}^{\#}} \mathrm{cl}\left(\bigcup_{t^{\prime} \in B} \Gamma_{t^{\prime}}\right),
\end{aligned}
$$

where $\mathscr{H}_{t}^{\#}=\left\{B \subset \mathbb{T} \mid B \cap O \neq \emptyset \forall O \in \mathscr{H}_{t}\right\}$ is the grill of $\mathscr{H}_{t}$; see [4]. The mapping $\Gamma$ is outer semicontinuous or inner semicontinuous, respectively, if

$$
\begin{array}{cc}
(\limsup \Gamma)_{t} \subset \Gamma_{t} & \forall t, \\
\Gamma_{t} \subset(\liminf \Gamma)_{t} & \forall t .
\end{array}
$$

We refer to [16, Chapter 5] for a systematic treatment of these concepts in the finite dimensional case. The above limits can also be expressed as

$$
\begin{aligned}
(\limsup \Gamma)_{t} & =\left\{x \in X \mid \Gamma^{-1}(A) \in \mathscr{H}_{t}^{\#} \forall A \in \mathscr{H}_{x}^{o}\right\}, \\
(\liminf \Gamma)_{t} & =\left\{x \in X \mid \Gamma^{-1}(A) \in \mathscr{H}_{t} \forall A \in \mathscr{H}_{x}^{o}\right\},
\end{aligned}
$$

where $\Gamma^{-1}(A)=\left\{t \mid \Gamma_{t} \cap A \neq \emptyset\right\}$. In [4] this was stated for a metric space $X$; for general $X$, (2) can be verified analogously to the proof of Lemma 1 below. Moreover, $\Gamma$ is inner semicontinuous if and only if $\Gamma^{-1}(A)$ is an open set for every open $A \subset X$ [10. Proposition 2.1]. This is taken as the definition of lower semicontinuity in [10].

Given a strictly positive countably additive measure $\mu$ on the Borel $\sigma$-algebra $\mathscr{B}(\mathbb{T})$, we define $\mathscr{H}_{t}^{\mu}=\left\{B \in \mathscr{B}(\mathbb{T}) \mid \exists O \in \mathscr{H}_{t}: \mu(B \cap O)=\mu(O)\right\}$ and

$$
(\mu-\liminf \Gamma)_{t}=\bigcap_{B \in \mathscr{H}_{t}^{\mu \#}} \mathrm{cl}\left(\bigcup_{t^{\prime} \in B} \Gamma_{t^{\prime}}\right),
$$


where $\mathscr{H}_{t}^{\mu \#}=\left\{B \in \mathscr{B}(\mathbb{T}) \mid B \cap O \neq \emptyset \forall O \in \mathscr{H}_{t}^{\mu}\right\}$. It is easily verified that

$$
\mathscr{H}_{t}{ }^{\mu \#}=\left\{B \in \mathscr{B}(\mathbb{T}) \mid \mu(B \cap O)>0 \forall O \in \mathscr{H}_{t}\right\} .
$$

We have $\mathscr{H}_{t} \subset \mathscr{H}_{t}^{\mu \#} \subset \mathscr{H}_{t}^{\#}$ and

$$
(\liminf \Gamma)_{t} \subseteq(\mu-\liminf \Gamma)_{t} \subseteq(\limsup \Gamma)_{t}
$$

All these limits are closed and they are invariant under the image closure. Here the image closure of $\Gamma$ is defined as $\mathrm{cl}_{t}$ for every $t$. Moreover, $\mu$-liminf is invariant under equivalent changes of measure.

Example 1. Assume that $X$ is Hausdorff and that $w: \mathbb{T} \rightarrow X$ has a continuous modification, i.e., there is a continuous $y: \mathbb{T} \rightarrow X$ such that $y_{t}=w_{t} \mu$-almost everywhere. Since $y$ is continuous, we have liminf $y_{t}=\limsup y_{t}$ so that by (3), $\mu$-liminf $y_{t}=y_{t}$ (we identify y with $t \mapsto\left\{y_{t}\right\}$ ). By Lemma 2 below, we get $\mu$-liminf $w_{t}=y_{t}$.

We say that $\Gamma$ is outer regular in measure or outer $\mu$-regular if

$$
(\mu-\liminf \Gamma)_{t} \subseteq \operatorname{cl} \Gamma_{t} \quad \forall t
$$

Outer regularity in measure is invariant under the image closure and under equivalent changes of measure. The sets of continuous selections and continuous essential selections of $\Gamma$ will be denoted by $C(\mathbb{T} ; \Gamma)$ and $C(\mathbb{T}, \mu ; \Gamma)$, respectively.

Theorem 1. If $\Gamma$ is closed-valued and outer $\mu$-regular, then

$$
C(\mathbb{T}, \mu ; \Gamma)=C(\mathbb{T} ; \Gamma) \text {. }
$$

Proof. Let $y \in C(\mathbb{T}, \mu ; \Gamma)$, and let $N \in \mathscr{B}(\mathbb{T})$ be a $\mu$-null set such that $\left\{t^{\prime} \mid y_{t^{\prime}} \notin \Gamma_{t^{\prime}}\right\} \subset$ $N$. Let $B \in \mathscr{H}_{t}^{\mu \#}$. Since $(B \backslash N) \cap O_{t} \neq \emptyset$ for every $O_{t} \in \mathscr{H}_{t}$, there is a sequence $\left(t^{v}\right)_{v=1}^{\infty}$ in $B \backslash N$ such that $t^{v} \rightarrow t$. Since $y_{t^{v}} \in \Gamma_{t^{v}}$ for every $v$, and since $y$ is continuous, we get $y_{t} \in \operatorname{cl}\left(\bigcup_{t^{\prime} \in B} \Gamma_{t^{\prime}}\right)$. Because this holds for every $B \in \mathscr{H}_{t}^{\mu \#}$, we have $y_{t} \in(\mu \text {-liminf } \Gamma)_{t}$. Since $\Gamma$ is outer $\mu$-regular, we get $y \in C(\mathbb{T} ; \Gamma)$. The opposite inclusion $C(\mathbb{T} ; \Gamma) \subseteq$ $C(\mathbb{T}, \mu ; \Gamma)$ is trivial.

Remark 1. Theorem 1 and the other results below hold for non-closed mappings with appropriate reformulations using image closures.

Section 2.1 will be concerned with properties of $\mu$-liminf which are applied in Section 2.2 to prove a converse of Theorem 1 . Our aim is to show that outer $\mu$-regularity is also a necessary condition in Theorem 1

Remark 2. Analogously to (1), one can define

$$
(\mu-\limsup \Gamma)_{t}=\bigcap_{B \in \mathscr{H}_{t}^{\mu}} \mathrm{cl}\left(\bigcup_{t^{\prime} \in B} \Gamma_{t^{\prime}}\right) .
$$

However, we do not analyze this limit concept here. 


\subsection{Properties of $\mu$-liminf}

The following result is comparable with (2). Recall that $\Gamma$ is measurable if $\Gamma^{-1}(A) \in$ $\mathscr{B}(\mathbb{T})$ for every open $A \subseteq X$.

Lemma 1. If $\Gamma$ is measurable, then

$$
(\mu-\liminf \Gamma)_{t}=\left\{x \in X \mid \Gamma^{-1}(A) \in \mathscr{H}_{t}^{\mu} \forall A \in \mathscr{H}_{x}^{o}\right\} .
$$

Proof. Assuming that there is an $A \in \mathscr{H}_{x}^{o}$ such that $\mu\left(O_{t} \cap \Gamma^{-1}(A)\right)<\mu\left(O_{t}\right)$ for all $O_{t} \in \mathscr{H}_{t}$, we have $\left(\Gamma^{-1}(A)\right)^{C} \in \mathscr{H}_{t}^{\mu \#}$, which implies $x \notin(\mu \text {-liminf } \Gamma)_{t}$, because $x \notin$ $\operatorname{cl}\left(\bigcup_{t \in\left(\Gamma^{-1}(A)\right)^{c}} \Gamma_{t}\right)$.

Assuming that $\Gamma^{-1}(A) \in \mathscr{H}_{t}^{\mu}$ for all $A \in \mathscr{H}_{x}^{o}$, we have $\operatorname{cl}\left(\bigcup_{t \in B} \Gamma_{t}\right) \cap A \neq \emptyset$ for all $B \in \mathscr{H}_{t}^{\mu \#}$ and $A \in \mathscr{H}_{x}^{o}$, which implies

$$
\{x\} \subseteq \bigcap_{B \in \mathscr{H}_{t}^{\mu \#}}\left[\bigcap_{A \in \mathscr{H}_{x}^{o}} \mathrm{cl}\left(\bigcup_{t \in B} \Gamma_{t}\right) \cap A\right] \subset(\mu-\liminf \Gamma)_{t} .
$$

The following lemma shows that $\mu$-liminf $\Gamma$ is invariant under changes of $\Gamma$ on $\mu$-null sets.

Lemma 2. If $\Gamma: \mathbb{T} \rightrightarrows X$ and $\tilde{\Gamma}: \mathbb{T} \rightrightarrows X$ are closed-valued and $\Gamma_{t}=\tilde{\Gamma}_{t} \mu$-a.e., then

$$
\mu-\liminf \Gamma_{t}=\mu-\liminf \tilde{\Gamma}_{t} \quad \forall t .
$$

Proof. There is a Borel $\mu$-null set $N$ such that $\Gamma_{t}=\tilde{\Gamma}_{t}$ for every $t \in N^{C}$. Since, for all $B \in \mathscr{B}(\mathbb{T})$, we have that $B \cap N^{C} \in \mathscr{H}_{t}^{\mu \#}$ if and only if $B \in \mathscr{H}_{t}^{\mu \#}$, we get

$$
\begin{aligned}
\bigcap_{B \in \mathscr{H}_{t}^{\mu \#}} \mathrm{cl}\left(\bigcup_{t^{\prime} \in B} \Gamma_{t^{\prime}}\right) & \subseteq \bigcap_{B \in \mathscr{H}_{t}^{\mu \#}}\left\{\mathrm{cl}\left(\bigcup_{t^{\prime} \in B \cap N^{C}} \Gamma_{t^{\prime}}\right) \mid B \cap N=\emptyset\right\} \\
& =\bigcap_{B \in \mathscr{H}_{t}^{\mu \#}} \mathrm{cl}\left(\bigcup_{t^{\prime} \in B \cap N^{C}} \Gamma_{t^{\prime}}\right) \\
& \subseteq \bigcap_{B \in \mathscr{H}_{t}^{\mu \#}} \mathrm{cl}\left(\bigcup_{t^{\prime} \in B} \Gamma_{t^{\prime}}\right)
\end{aligned}
$$

which gives

$$
\mu-\liminf \Gamma_{t}=\bigcap_{B \in \mathscr{H}_{t}^{\mu \#}} \mathrm{cl}\left(\bigcup_{t^{\prime} \in B \cap N^{C}} \Gamma_{t^{\prime}}\right) .
$$

This implies that $\mu-\liminf \Gamma_{t}=\mu-\liminf \tilde{\Gamma}_{t}$ for all $t$. 
Recall that $\mathbb{T}$ is Lindelöf if every open cover of $\mathbb{T}$ has a countable subcover, and that $\mathbb{T}$ is strongly Lindelöf if every subspace of $\mathbb{T}$ is Lindelöf. When $X$ is a normed space, $\mathbb{B}(x, r)$ denotes the open ball with center $x$ and radius $r$, and $d(A, B)=\inf \left\{\left\|x-x^{\prime}\right\| \mid\right.$ $\left.x \in A, x^{\prime} \in B\right\}$ denotes the distance between two sets $A$ and $B$. A convex-valued $\Gamma$ is solid if it is closed-valued and int $\Gamma_{t} \neq \emptyset$ for all $t$.

Proposition 1. Assume that $\mathbb{T}$ is strongly Lindelöf, $X=\mathbb{R}^{d}$ and that $\Gamma$ is measurable and closed-valued.

(A) If $\Gamma$ is convex-valued, then $t \mapsto \mu$-liminf $\Gamma_{t}$ is convex-valued.

(B) If $\Gamma$ is inner semicontinuous solid convex-valued, then $\mu$-liminf $\Gamma$ is inner semicontinuous solid convex-valued, $\mu$-liminf $\Gamma_{t}=\mu$-liminf $\Gamma_{t} \mu$-a.e. and

$$
\mu-\liminf (\mu-\liminf \Gamma)=\mu-\liminf \Gamma \text {. }
$$

Proof. Let $\left(A^{v}\right)_{v=1}^{\infty}$ be a countable open base for the topology on $X$. For every $v$ and $t$ with $t \in(\mu \text {-liminf } \Gamma)^{-1}\left(A^{v}\right)$, there is, by Lemma11 an open $O_{t}^{v} \in \mathscr{H}_{t}$ and a $\mu$-null set $N_{t}^{v} \in \mathscr{B}(\mathbb{T})$ such that $O_{t}^{v} \backslash \Gamma^{-1}\left(A^{v}\right)=N_{t}^{v}$. Since $\mathbb{T}$ is strongly Lindelöf, there is a countable $\mathscr{J}^{v}$ (here $\mathscr{J}^{v}=\emptyset$ if $\left.(\mu \text {-liminf } \Gamma)^{-1}\left(A^{v}\right)=\emptyset\right)$ for which $(\mu \text {-liminf } \Gamma)^{-1}\left(A^{v}\right) \subset$ $\bigcup_{t \in \mathscr{J} v} O_{t}^{v}$. Let

$$
N=\bigcup_{v, t \in \mathscr{J}^{v}} N_{t}^{v},
$$

which is a $\mu$-null-set.

Let $t^{\prime}$ be such that $\mu$-liminf $\Gamma_{t^{\prime}} \nsubseteq \Gamma_{t^{\prime}}$. There is an $A^{v}$ such that $\mu-\liminf \Gamma_{t^{\prime}} \cap A^{v} \neq \emptyset$ but $\Gamma_{t^{\prime}} \cap A^{v}=\emptyset$. Choose $t \in \mathscr{J}^{v}$ such that $t^{\prime} \in O_{t}^{v}$. Since $O_{t}^{v} \backslash \Gamma^{-1}\left(A^{v}\right)=N_{t}^{v}$ and since $t^{\prime} \notin \Gamma^{-1}\left(A^{v}\right)$, we have $t^{\prime} \in N$. Thus $\mu$-liminf $\Gamma_{t} \subseteq \Gamma_{t}$ for all $t \in N^{C}$.

Assume now that $\Gamma$ is convex-valued. Let $x^{1}, x^{2} \in(\mu \text {-liminf } \Gamma)_{t}$ and $\alpha \in[0,1]$, and denote $\bar{x}=\alpha x^{1}+(1-\alpha) x^{2}$. Let $A \in \mathscr{H}_{\bar{x}}^{o}$ be convex. By Lemma 1 and the construction of $N$, there is $O_{t}^{1}, O_{t}^{2} \in \mathscr{H}_{t}$ such that $\Gamma^{-1}\left(A-\bar{x}+x^{1}\right)=O_{t}^{1} \backslash N$ and $\Gamma^{-1}\left(A-\bar{x}+x^{2}\right)=$ $O_{t}^{2} \backslash N$. For any $t^{\prime} \in O_{t}^{2} \cap O_{t}^{1} \backslash N$, there is an $x_{t^{\prime}}^{1} \in \Gamma_{t^{\prime}} \cap\left(A-\bar{x}+x^{1}\right)$ and an $x_{t^{\prime}}^{2} \in \Gamma_{t^{\prime}} \cap$ $\left(A-\bar{x}+x^{2}\right)$ so that $\alpha x_{t^{\prime}}^{1}+(1-\alpha) x_{t^{\prime}}^{2} \in \Gamma_{t^{\prime}} \cap A$, which implies $\Gamma^{-1}(A) \in \mathscr{H}_{t}^{\mu}$. Since this holds for every convex $A \in \mathscr{H}_{\bar{x}}^{o}$, and since every element of $\mathscr{H}_{\bar{x}}^{o}$ contains some convex $A \in \mathscr{H}_{\bar{x}}^{o}$, we get, by Lemma1, that $\bar{x} \in \mu-\liminf \Gamma_{t}$.

Assume now that $\Gamma$ is inner semicontinuous solid convex-valued. By inner semicontinuity of $\Gamma$, (3) and by $\mu$-liminf $\Gamma_{t} \subseteq \Gamma_{t}$ for all $t \in N^{C}$, we get

$$
\mu-\liminf \Gamma_{t}=\Gamma_{t} \quad \forall t \in N^{C} .
$$

Thus, by Lemma2, we have $\mu-\liminf (\mu-\liminf \Gamma)=\mu-\liminf \Gamma$.

By (3), int $\mu$-liminf $\Gamma_{t} \neq \emptyset$ for all $t$. Since $\mu$-liminf $\Gamma$ is convex-valued, to prove inner semicontinuity of $\mu$-liminf $\Gamma$, it suffices to show that $\bar{x} \in \liminf \mu$-liminf $\Gamma_{\bar{t}}$ whenever $\bar{x} \in \operatorname{int} \mu$-liminf $\Gamma_{\bar{t}}$. Let $\left(x^{i}\right)_{i=1}^{d}$ be an orthonormal basis in $\mathbb{R}^{d}$ and $\varepsilon>0$ be such that $\bar{x} \pm \varepsilon x^{i} \in \mu$-liminf $\Gamma_{\bar{t}}$ for all $i$. Since there are finitely many $x^{i}$, there is, by Lemma 1 , an open $O_{\bar{t}} \in \mathscr{H}_{\bar{t}}$ such that $\Gamma_{t} \cap \mathbb{B}\left(\bar{x} \pm \varepsilon x^{i}, \varepsilon / 2\right) \neq \emptyset \mu$-a.e. on $O_{\bar{t}}$ for all $x^{i}$. Since $\Gamma$ is convex-valued, we have $\bar{x} \in \Gamma_{t} \mu$-a.e. on $O_{\bar{t}}$. Therefore, by Lemma $1 \bar{x} \in \mu$ - $\liminf \Gamma_{t}$ for all $t \in O_{\bar{t}}$ and consequently $\bar{x} \in \liminf \mu-\liminf \Gamma_{\bar{t}}$. 
The following example illustrates that, without convexity, inner semicontinuity is not necessarily preserved under $\mu$-liminf.

Example 2. Let $\mathbb{T}=[0,1]$ be equipped with the standard topology, $\mu$ be the Lebesque measure and let $\tilde{\Gamma}_{t}=\left\{2^{-n}\right\}$ for $t \in\left(2^{-(n+1)}, 2^{-n}\right)$ and $n \in \mathbb{N}$. Let $\Gamma_{t}=\tilde{\Gamma}_{t} \cup[1,2]$ so that $\Gamma$ is inner semicontinuous closed nonempty-valued. It follows from Lemma 1 that $\mu$-liminf $\Gamma_{0}=\{0\} \cup[1,2]$ but $\mu$-liminf $\Gamma_{t}=[1,2]$ whenever $t=2^{-n}$ for some $n \in \mathbb{N}$. In particular, $t \mapsto \mu$-liminf $\Gamma_{t}$ is not inner semicontinuous at the origin.

A closed convex $\mathbb{R}^{d}$-valued $\Gamma$ is fully lower semicontinuous if it is inner semicontinuous solid-valued and $x \in \operatorname{cl} \Gamma_{t}$ whenever there exist $A \in \mathscr{H}_{x}$ and $O \in \mathscr{H}_{t}$ such that $\left\{t \in O \mid A \subset \Gamma_{t}\right\}$ is dense in $O$. In [14, Theorem 5] Rockafellar uses full lower semicontinuity to prove an explicit expression for the convex conjugate of an integral functional on continuous functions.

Lemma 3. Assume that $\mathbb{T} \subseteq \mathbb{R}^{n}$. If $\Gamma$ is closed convex $\mathbb{R}^{d}$-valued and fully lower semicontinuous, then $\Gamma$ is outer regular in measure.

Proof. By Proposition 1, the mapping $\mu$-liminf $\Gamma$ is inner semicontinuous solid convexvalued. It suffices to show that, for any $t \in \mathbb{T}$ and $x \in \operatorname{int} \mu-\liminf \Gamma_{t}$, we have $x \in \operatorname{cl} \Gamma_{t}$.

Let $\varepsilon>0$ be such that $\operatorname{cl} \mathbb{B}(x, \varepsilon) \subset \mu$-liminf $\Gamma_{t}$. By [4, Proposition 1.6], there is $O_{t} \in$ $\mathscr{H}_{t}$ such that $\operatorname{cl} \mathbb{B}(x, \varepsilon) \subset \mu$-liminf $\Gamma_{t}+\operatorname{cl} \mathbb{B}(0, \varepsilon / 2)$ for all $t \in O_{t}$. Thus $\mathbb{B}(x, \varepsilon / 2) \subset$ $\mu$-liminf $\Gamma_{t}$ for all $t \in O_{t}$. By Proposition $1, \mu$-liminf $\Gamma_{t}=\Gamma_{t} \mu$-a.e. so that there is a $\mu$-null set $N \in \mathscr{B}(\mathbb{T})$ such that $\mathbb{B}(x, \varepsilon / 2) \subset \Gamma_{t}$ for all $t \in O_{t} \cap N^{C}$. Since $\Gamma$ is fully lower semicontinuous and $\left\{t \in O_{t} \mid \mathbb{B}(x, \varepsilon / 2) \subset \Gamma_{t}\right\}$ is dense in $O_{t}$, we get $x \in \operatorname{cl} \Gamma_{t}$.

The following example demonstrates that there are inner semicontinuous solid convex-valued mappings which are outer $\mu$-regular but not fully lower semicontinuous.

Example 3. Let $\mathbb{T} \subseteq \mathbb{R}^{n}$ be open and $S \subset \mathbb{T}$ be a $(n-1)$-dimensional closed set such that $\mathbb{T} \backslash S$ is dense in $\mathbb{T}$. Let $H$ be the restriction of $(n-1)$-dimensional Hausdorff measure to $S$, and assume that $\operatorname{supp}(H)=S$ (see, e.g., [2]). Let $\mu=\lambda+H$, where $\lambda$ is the Lebesque measure on $\mathbb{T}$. Define

$$
\Gamma_{t}= \begin{cases}\operatorname{cl} \mathbb{B}\left(0, r_{2}\right) & \text { if } t \notin S, \\ \operatorname{cl} \mathbb{B}\left(0, r_{1}\right) & \text { if } t \in S,\end{cases}
$$

where $0 \leq r_{1}<r_{2} \leq+\infty$. Since $S$ is a closed set, $\Gamma^{-1}(A)$ is open for every open $A$, and therefore $\Gamma$ is inner semicontinuous. To check outer $\mu$-regularity of $\Gamma$, it suffices to consider $t \in S$ and $x \notin \mathrm{cl} \mathbb{B}\left(0, r_{1}\right)$. Let $A \in \mathscr{H}_{x}^{o}$ be such that $\operatorname{cl} \mathbb{B}\left(0, r_{1}\right) \cap A=\emptyset$. Since $\operatorname{supp}(H)=S$, we have $\mu\left(\Gamma^{-1}(A) \cap O\right) \leq \lambda(O)<\mu(O)$ for every $O \in \mathscr{H}_{t}$ so that, by Lemma $1 x \notin \mu$-liminf $\Gamma_{t}$. Thus $\mu$-liminf $\Gamma_{t} \subseteq \operatorname{cl} \Gamma_{t}$ for all $t$ and $\Gamma$ is outer $\mu$-regular.

Assume now that $0<r_{1}$. Let $x \in \mathbb{B}\left(0, r_{2}\right)$. There is an $A \subset \mathscr{H}_{x}$ such that $A \subset$ $\operatorname{cl} \mathbb{B}\left(0, r_{2}\right)$. Thus $\left\{t \in \mathbb{T} \mid A \subset \Gamma_{t}\right\}$ is dense in $\mathbb{T}$, because it contains $\mathbb{T} \backslash S$. However, $x \notin$ $\operatorname{cl} \Gamma_{t}$ whenever $x \notin \operatorname{cl} \mathbb{B}\left(0, r_{1}\right)$ and $t \in S$. Therefore, $\Gamma$ is not fully lower semicontinuous though it is inner semicontinuous solid convex-valued. 


\subsection{Necessity of outer regularity in measure}

In this section we show that, for inner semicontinuous solid convex $\mathbb{R}^{d}$-valued mappings, the condition $C(\mathbb{T}, \mu ; \Gamma)=C(\mathbb{T} ; \Gamma)$ in Theorem 1 is necessary for outer $\mu$ regularity. The proof is based on [10, Lemma 5.2], which says that if $\mathbb{T}$ is a perfectly normal $T_{1}$-space, $X$ is a separable Banach space and $\Gamma$ is an inner semicontinuous closed convex nonempty-valued mapping, then there exists a sequence $\left(y^{v}\right)_{v=1}^{\infty} \subset$ $C(\mathbb{T} ; \Gamma)$ such that $\left(y_{t}^{v}\right)_{v}^{\infty}$ is dense in $\Gamma_{t}$ for every $t$. Such a sequence is usually referred to as a Michael representation of $\Gamma$.

Recall that a topological space is $T_{1}$ if for every distinct points $t$ and $t^{\prime}$ there is an $O_{t} \in \mathscr{H}_{t}$ with $t^{\prime} \notin O_{t}$. The space is normal if for every disjoint closed sets $B$ and $B^{\prime}$ there are disjoint open sets $O$ and $O^{\prime}$ such that $B \subset O$ and $B^{\prime} \subset O^{\prime}$. The space $\mathbb{T}$ is perfectly normal if it is normal and every closed set is a countable intersection of open sets.

Theorem 2. Assume that $\mathbb{T}$ is a Lindelöf perfectly normal $T_{1}$-space. An inner semicontinuous solid convex $\mathbb{R}^{d}$-valued mapping $\Gamma$ is outer $\mu$-regular if and only if $C(\mathbb{T}, \mu ; \Gamma)=$ $C(\mathbb{T} ; \Gamma)$.

Proof. Assuming that $\Gamma$ is outer $\mu$-regular, Theorem 1 implies that $C(\mathbb{T}, \mu ; \Gamma)=C(\mathbb{T} ; \Gamma)$. Assume that $C(\mathbb{T}, \mu ; \Gamma)=C(\mathbb{T} ; \Gamma)$. We use the fact that a Lindelöf perfectly normal space is strongly Lindelöf (see [7, p. 194]). By Proposition $1, t \mapsto \mu-\liminf \Gamma_{t}$ is an inner semicontinuous closed convex nonempty-valued mapping. By [10, Lemma 5.2], there is a $\left(y^{v}\right)_{v=1}^{\infty} \subseteq C(\mathbb{T} ; \mu$-liminf $\Gamma)$ such that $\left(y_{t}^{v}\right)_{v=1}^{\infty}$ is dense in $\mu$-liminf $\Gamma_{t}$ for every $t$. Thus

$$
\mu-\liminf \Gamma_{t}=\operatorname{cl}\left\{y_{t}^{v} \mid v \geq 1\right\} \subseteq \Gamma_{t} \quad \forall t,
$$

where the inclusion follows from Proposition 1, because now $\mu$-liminf $\Gamma_{t}=\Gamma_{t} \mu$-a.e. implies $C(\mathbb{T} ; \mu$-liminf $\Gamma)=C(\mathbb{T}, \mu ; \Gamma)=C(\mathbb{T} ; \Gamma)$. This shows that $\Gamma$ is outer $\mu$-regular.

Remark 3. Let $\bar{\Gamma}$ be the image closure of $\Gamma$. The proof of Theorem 2 actually shows that if $t \mapsto \mu$-liminf $\Gamma$ is inner semicontinuous convex nonempty-valued, then $\Gamma$ is outer $\mu$-regular if and only if $C(\mathbb{T}, \mu ; \bar{\Gamma})=C(\mathbb{T} ; \bar{\Gamma})$. In this case the last inclusion in the proof follows from $\mu$-liminf $\Gamma_{t} \subseteq \operatorname{cl} \Gamma_{t} \mu$-a.e. (see the proof of Proposition 1 ).

Without convexity or inner semicontinuity the necessity in Theorem 2 does not hold in general. Indeed, in either situation continuous selections need not exist at all.

\section{Applications to conjugates of integral functionals}

From now on we will assume that $\mathbb{T}$ is a Lindelöf perfectly normal $T_{1}$-space and $X=$ $\mathbb{R}^{d}$. Let $h$ be a convex normal integrand on $\mathbb{T} \times \mathbb{R}^{d}$, i.e., $h$ is an extended real-valued function and $t \mapsto$ epi $h_{t}=\left\{(x, \alpha) \mid h_{t}(x) \leq \alpha\right\}$ is closed convex-valued and measurable from $\mathbb{T}$ to $\mathbb{R}^{d} \times \mathbb{R}$. By [16, Proposition 14.28], $t \mapsto h_{t}\left(w_{t}\right)$ is measurable whenever $w: \mathbb{T} \rightarrow \mathbb{R}^{d}$ is measurable so that the integral functional

$$
I_{h}(y)=\int_{\mathbb{T}} h_{t}\left(y_{t}\right) d \mu_{t}
$$


is well-defined on $C=C\left(\mathbb{T} ; \mathbb{R}^{d}\right)$. Here and in what follows the integral of a measurable function is defined as $+\infty$ unless the positive part is integrable.

Let $C_{b}=C_{b}\left(\mathbb{T} ; \mathbb{R}^{d}\right)$ be the space of bounded continuous functions and $M_{b}$ be the space of $\mathbb{R}^{d}$-valued finite Radon measures on $\mathbb{T}$. The bilinear form

$$
\langle y, \theta\rangle=\int_{\mathbb{T}} y_{t} d \theta_{t}
$$

puts the spaces $C_{b}$ and $M_{b}$ in separating duality. Indeed, it follows from [5, p.71] that $C_{b}$ separates the points in $M_{b}$, whereas it is evident that $M_{b}$ separates the points of $C_{b}$.

Our aim is to study the conjugate

$$
I_{h}^{*}(\theta)=\sup \left\{\langle y, \theta\rangle-I_{h}(y)\right\} .
$$

In [14, Theorem 5] Rockafellar gave conditions under which the conjugate of $I_{h}$ can be expressed in terms of the conjugate of $h$ as

$$
J_{h^{*}}(\theta)=\int_{\mathbb{T}} h_{t}^{*}\left(\left(\frac{d \theta}{d \mu}\right)_{t}\right) d \mu_{t}+\int_{\mathbb{T}}\left(h^{*}\right)_{t}^{\infty}\left(\left(\frac{d \theta^{s}}{d\left|\theta^{s}\right|}\right)_{t}\right) d\left|\theta^{s}\right|_{t},
$$

where $\theta^{s}$ is the singular part of $\theta \in M_{b}$ with respect to $\mu$. Here the conjugate of $h$ is defined by $h_{t}^{*}(v)=\sup _{x \in \mathbb{R}^{d}}\left\{x \cdot v-h_{t}(x)\right\}$ and $\left(h_{t}^{*}\right)^{\infty}$ denotes the recession function of $h_{t}^{*}$. That is, $\left(h_{t}^{*}\right)^{\infty}$ is defined by

$$
\left(h_{t}^{*}\right)^{\infty}(v)=\sup _{\alpha>0} \frac{h_{t}^{*}(\alpha v+\bar{v})-h_{t}^{*}(\bar{v})}{\alpha}
$$

where $\bar{v} \in \operatorname{dom} h_{t}^{*}=\left\{v \in \mathbb{R}^{d} \mid h_{t}^{*}(v)<\infty\right\}$; see [13, Chapter 8]. We will use the techniques from [6] to generalize Rockafellar's result and to prove a result for integral functionals on functions of bounded variation. For other related results, see, e.g., [1, 2, 3, 6, 14, 18] and references therein.

We denote the relative interior of a set $A \subset \mathbb{R}^{d}$ by $\operatorname{rint} A$. Recall that $\mu$ is $\sigma$-finite if $\mathbb{T}$ is a countable union of sets with finite $\mu$-measure.

Theorem 3. Assume that $\mu$ is a $\sigma$-finite Radon measure, dom $h$ is inner semicontinuous, $\operatorname{dom} J_{h^{*}} \neq \emptyset, C_{b}(\mathbb{T} ; \operatorname{rint} \operatorname{dom} h) \cap \operatorname{dom} I_{h} \neq \emptyset$ and that for every $y \in C(\mathbb{T} ; \operatorname{rint} \mu$-liminf $\operatorname{dom} h)$ and for every $t$ there exists $O_{t} \in \mathscr{H}_{t}$ such that

$$
\int_{O_{t}}\left|h_{t}\left(y_{t}\right)\right| d \mu_{t}<\infty
$$

If $\operatorname{dom} h$ is outer $\mu$-regular, then $I_{h}$ and $J_{h^{*}}$ are conjugates of each other. If int $\operatorname{dom} h_{t} \neq$ $\emptyset$ for all $t$ and if $I_{h}$ and $J_{h^{*}}$ are conjugates of each other, then dom $h$ is outer $\mu$-regular.

\footnotetext{
${ }^{2}$ A measure $\theta$ is Radon if it is countably additive and $|\theta|(B)=\sup \{|\theta|(K) \mid K \subseteq B, K$ is compact $\}$ for every $B \in \mathscr{B}(\mathbb{T})$.
} 
Proof. We have that

$$
\begin{aligned}
J_{h^{*}}^{*}(y)= & \sup _{\theta \in M_{b}}\left\{\int_{\mathbb{T}} y_{t} d \theta_{t}-J_{h^{*}}(\theta)\right\} \\
= & \sup _{\theta^{\prime} \in L^{1}\left(\mathbb{T}, \mu ; \mathbb{R}^{d}\right)}\left\{\int_{\mathbb{T}} y_{t} \cdot \theta_{t}^{\prime} d \mu_{t}-I_{h^{*}}\left(\theta^{\prime}\right)\right\} \\
& +\sup _{\theta \in M_{b}, \theta^{s^{\prime}} \in L^{1}\left(\mathbb{T},\left|\theta^{s}\right| ; \mathbb{R}^{d}\right)}\left\{\int_{\mathbb{T}} y_{t} \cdot \theta_{t}^{s^{\prime}} d\left|\theta^{s}\right|_{t}-\int_{\mathbb{T}}\left(h_{t}^{*}\right)^{\infty}\left(\theta_{t}^{s^{\prime}}\right) d\left|\theta^{s}\right|_{t}\right\} \\
= & \begin{cases}I_{h}(y) & \text { if } y_{t} \in \operatorname{cldom} h_{t} \forall t, \\
+\infty & \text { otherwise. }\end{cases}
\end{aligned}
$$

Above the second equality follows from the positive homogeneity of $\left(h_{t}^{*}\right)^{\infty}[13$, Theorem 8.5], and the third equality follows by first applying [16. Theorem 14.60] on the second and the third line, where one uses the fact that the indicator function $\delta_{\mathrm{cldom}} h_{t}$ is the conjugate of $\left(h_{t}^{*}\right)^{\infty}$ [13, Theorem 13.3]; and then taking supremum over all purely atomic finite measures which are singular with respect to $\mu$. On the other hand, since $\operatorname{dom} J_{h^{*}} \neq \emptyset$, there is a $\bar{\theta} \in \operatorname{dom} J_{h^{*}}$ which is absolutely continuous with respect to $\mu$. Therefore, for all $w \in L^{\infty}\left(\mathbb{T}, \mu ; \mathbb{R}^{d}\right)$,

$$
\int_{\mathbb{T}} h_{t}\left(w_{t}\right) d \mu_{t} \geq \int_{\mathbb{T}}\left[w_{t} \cdot\left(\frac{d \bar{\theta}}{d \mu}\right)_{t}-h_{t}^{*}\left(\left(\frac{d \bar{\theta}}{d \mu}\right)_{t}\right)\right] d \mu_{t}>-\infty .
$$

Assume first that $\operatorname{dom} h$ is outer $\mu$-regular. By Theorem $1 y_{t} \in \operatorname{cldom} h_{t}$ for every $t$ whenever $y \in \operatorname{dom} I_{h}$ so that (5) implies that $J_{h^{*}}^{*}=I_{h}$. Therefore, $I_{h}^{*} \leq J_{h^{*}}$ and consequently it suffices to show that $I_{h}^{*} \geq J_{h^{*}}$.

Since $\left|\theta^{s}\right|$ and $\mu$ are singular, there is a $\bar{B} \in \mathscr{B}(T)$ such that $\mu\left(\bar{B}^{C}\right)=\left|\theta^{s}\right|(\bar{B})=0$. We define

$$
\bar{h}_{t}(x)= \begin{cases}-x \cdot\left(\frac{d \theta}{d \mu}\right)_{t}+h_{t}(x) & \text { if } t \in \bar{B} \\ -x \cdot\left(\frac{d \theta^{s}}{d \mid \theta^{s}}\right)_{t}+\delta_{\text {cldom } h_{t}}(x) & \text { if } t \in \bar{B}^{C},\end{cases}
$$

which is a normal integrand (see [16, Chapter 14]) with $\operatorname{cldom} \bar{h}_{t}=\operatorname{cldom} h_{t}$ for all $t$. Since $y_{t} \in \operatorname{cldom} h_{t}$ for all $t$ whenever $I_{h}(y)<\infty$ and since, by (6), $I_{h}(y)>-\infty$ for all $y$, we have

$$
\begin{aligned}
I_{h}^{*}(\theta)=-\inf _{y \in C_{b}}\left\{\int_{\bar{B}}\left[-y_{t} \cdot\left(\frac{d \theta}{d \mu}\right)_{t}+h_{t}\left(y_{t}\right)\right] d \mu_{t}\right. \\
\left.\quad+\int_{\bar{B}^{C}}\left[-y_{t} \cdot\left(\frac{d \theta^{s}}{d\left|\theta^{s}\right|}\right)_{t}+\delta_{\mathrm{cldom} h_{t}}\left(y_{t}\right)\right] d\left|\theta^{s}\right|_{t}\right\} \\
=-\inf _{y \in C_{b}} I_{\bar{h}}^{\theta}(y),
\end{aligned}
$$

where we defined $\mu^{\theta}=\mu+\left|\theta^{s}\right|$ and $I_{\bar{h}}^{\theta}(w)=\int_{\mathbb{T}} \bar{h}_{t}\left(w_{t}\right) d \mu_{t}^{\theta}$ for any measurable $w$ : 
$\mathbb{T} \rightarrow \mathbb{R}^{d}$. By [16, Theorem 14.60],

$$
\begin{aligned}
\inf _{w \in L^{\infty}\left(\mathbb{T}, \mu^{\theta} ; \mathbb{R}^{d}\right)} I_{\bar{h}}^{\theta}(w) & =\int_{\mathbb{T}} \inf _{x \in \mathbb{R}^{d}} \bar{h}_{t}(x) d \mu_{t}^{\theta} \\
& =-\int_{\mathbb{T}} h_{t}^{*}\left(\left(\frac{d \theta}{d \mu}\right)_{t}\right) d \mu_{t}-\int_{\mathbb{T}}\left(h_{t}^{*}\right)^{\infty}\left(\left(\frac{d \theta^{s}}{d\left|\theta^{s}\right|}\right)_{t}\right) d\left|\theta^{s}\right|_{t} \\
& =-J_{h^{*}}(\theta) .
\end{aligned}
$$

Let us show that we can restrict the infimum in the above expression to $w \in L^{\infty}\left(\mathbb{T}, \mu^{\theta} ; \mathbb{R}^{d}\right)$ with $w_{t} \in \operatorname{rint} \operatorname{dom} h_{t}$ for all $t$. Firstly, if $I_{\bar{h}}^{\theta}(w)<\infty$, then there is $\bar{w}$ such that $\bar{w}_{t}=w_{t}$ outside a Borel $\mu^{\theta}$-null set and $\bar{w}_{t} \in \operatorname{cldom} h_{t}$ for all $t$. Secondly, let $\bar{y} \in C_{b}(\mathbb{T}$; rint $\operatorname{dom} h) \cap$ $\operatorname{dom} I_{h}$ so that $w^{v}=\frac{1}{v} \bar{y}+\left(1-\frac{1}{v}\right) \bar{w}$ satisfies $w_{t}^{v} \in \operatorname{rint} \operatorname{dom} h_{t}$ for all $t$. It follows from

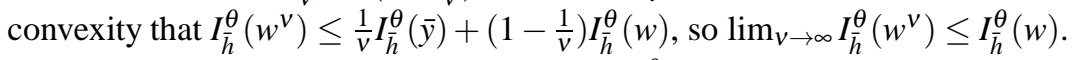

On the other hand, it follows from (6) that $I_{\bar{h}}^{\theta}(w)>-\infty$ for every $w \in L^{\infty}\left(\mathbb{T}, \mu^{\theta} ; \mathbb{R}^{d}\right)$. Therefore it suffices to show that, for every $w \in L^{\infty}\left(\mathbb{T}, \mu^{\theta} ; \mathbb{R}^{d}\right)$ with $I_{\bar{h}}^{\theta}(w)<\infty$ and $w_{t} \in \operatorname{rintdom} h_{t}$ for all $t$ and for every $\varepsilon>0$, there is $y \in C_{b}$ for which $I_{\bar{h}}^{\theta}(y) \leq I_{\bar{h}}^{\theta}(w)+\varepsilon$.

Since $\mu^{\theta}$ is Radon and since $I_{\bar{h}}^{\theta}(\bar{y})$ and $I_{\bar{h}}^{\theta}(w)$ are finite, there is, by Lusin's theorem (see, e.g., [5, Theorem 7.1.13]), an open $\bar{O} \subset \mathbb{T}$ such that $\int_{\bar{O}}\left(\left|\bar{h}_{t}\left(\bar{y}_{t}\right)\right|+\left|\bar{h}_{t}\left(w_{t}\right)\right|\right) d \mu^{\theta}<$ $\varepsilon / 3, \bar{O}^{C}$ is compact and $w$ is continuous relative to $\bar{O}^{C}$. The mapping

$$
\Gamma_{t}= \begin{cases}w_{t} & \text { if } t \in \bar{O}^{C} \\ \operatorname{rintdom} h_{t} & \text { if } t \in O\end{cases}
$$

is inner semicontinuous convex nonempty-valued so that, by [10, Theorem 3.1"'], there is a $y^{w} \in C$ with $y_{t}^{w}=w_{t}$ for all $t \in \bar{O}^{C}$ and $y_{t}^{w} \in \operatorname{rintdom} h_{t}$ for all $t$. Since $\bar{O}^{C}$ is compact, there is an open $O^{b} \supset \bar{O}^{C}$ such that $y^{w}$ is bounded on $O^{b}$.

Since, for all $t \in O^{b}$, there is an $O_{t} \in \mathscr{H}_{t}$ with $\int_{O_{t}}\left|\bar{h}_{t}\left(y_{t}^{w}\right)\right| d \mu_{t}^{\theta}<\infty$, and since $\bar{O}^{C}$ is compact, there is an open set $O^{\prime} \supset \bar{O}^{C}$ such that $B \mapsto \int_{O^{\prime} \cap B}\left|\bar{h}_{t}\left(y_{t}^{w}\right)\right| d \mu_{t}^{\theta}$ is a finite measure, which is also Radon (see [5, Lemma 7.1.11]). Therefore [8, Theorem 3.4] implies the existence of an open $O \supset \bar{O}^{C}$ with $\int_{O \backslash \bar{O}^{C}}\left|\bar{h}_{t}\left(y_{t}^{w}\right)\right| d \mu_{t}^{\theta}<\varepsilon / 3$.

Define $\hat{O}=O \cap O^{b}$. Since $\hat{O}$ and $\bar{O}$ form an open cover of $\mathbb{T}$ and since $\mathbb{T}$ is normal, there is, by [11, Theorem 36.1], a continuous partition of unity $(\hat{\alpha}, \bar{\alpha})$ subordinate to $(\hat{O}, \bar{O})$. We define $y=\hat{\alpha} y^{w}+\bar{\alpha} \bar{y}$ so that $y \in C_{b}$ and

$$
\begin{aligned}
\int_{\mathbb{T}} \bar{h}_{t}\left(y_{t}\right) d \mu_{t}^{\theta} & \leq \int_{\bar{O}^{C}} \bar{h}_{t}\left(w_{t}\right) d \mu_{t}^{\theta}+\int_{\hat{O} \backslash \overline{\bar{O}}} \hat{\alpha}_{t}\left|\bar{h}_{t}\left(y_{t}^{w}\right)\right| d \mu_{t}^{\theta}+\int_{\bar{O}} \bar{\alpha}\left|\bar{h}_{t}\left(\bar{y}_{t}\right)\right| d \mu_{t}^{\theta} \\
& \leq \int_{h_{t}}\left(w_{t}\right) d \mu_{t}^{\theta}+\varepsilon
\end{aligned}
$$

which finishes the proof of the sufficiency.

Assume now that int $\operatorname{dom} h_{t} \neq \emptyset$ for all $t, I_{h}^{*}=J_{h^{*}}$ and that $J_{h^{*}}^{*}=I_{h}$. We prove outer $\mu$-regularity of $\operatorname{dom} h$ using Theorem 2 Let $\tilde{y} \in C$ be such that $\tilde{y}_{t} \in \operatorname{cldom} h_{t} \mu$-a.e. By Proposition 1 and Theorem $1, \tilde{y} \in C(\mathbb{T} ; \mu$-liminfdom $h)$ so that, for every $v \geq 1$, by (3), the function $\tilde{y}^{v}=\frac{1}{v} \bar{y}+\left(1-\frac{1}{v}\right) \tilde{y}$ satisfies $\tilde{y}^{v} \in C(\mathbb{T}$, int $\mu$-liminfdom $h)$. 
Fix $v$ and $t$. There is an $O_{t} \in \mathscr{H}_{t}$ such that $\int_{O_{t}}\left|h_{t}\left(\tilde{y}_{t}^{v}\right)\right| d \mu_{t}<\infty$ and $\tilde{y}^{v}$ is bounded on $O_{t}$. Let $\left(\alpha^{1}, \alpha^{2}\right)$ be a continuous partition of unity subordinate to $\left(O_{t}, \mathbb{T} \backslash\{t\}\right)$. We define $y^{v}=\alpha^{1} \tilde{y}^{v}+\alpha^{2} \bar{y}$, which satisfies $y^{v} \in \operatorname{dom} I_{h}$. Therefore (5) implies that $y_{t^{\prime}}^{v} \in \operatorname{cldom} h_{t^{\prime}}$ for all $t^{\prime}$. Since $y_{t}^{v} \rightarrow \tilde{y}_{t}$, we have that $\tilde{y}_{t} \in \operatorname{cldom} h_{t}$. Since $t$ was arbitrary, $\tilde{y}_{t} \in \operatorname{cldom} h_{t}$ for all $t$. By Theorem 2 , dom $h$ is outer $\mu$-regular.

When $\mathbb{T} \subset \mathbb{R}^{n}$, the following corollary generalizes [14, Theorem 5] by relaxing full lower semicontinuity of $\operatorname{dom} h$; see Lemma3

Corollary 1. Assume that $\mathbb{T} \subset \mathbb{R}^{n}$ is compact, $\mu$ is a $\sigma$-finite Radon measure, dom $h$ is inner semicontinuous with int $\operatorname{dom} h_{t} \neq \emptyset$ for all $t$ and that $I_{h}$ is finite on

$$
\left\{y \in C \mid \exists r>0: \mathbb{B}\left(y_{t}, r\right) \subset \operatorname{dom} h_{t} \mu \text {-a.e. }\right\} .
$$

Then $\operatorname{dom} h$ is outer $\mu$-regular if and only if $I_{h}$ and $J_{h}^{*}$ are conjugates of each other. In this case int $\operatorname{dom} I_{h}=C(\mathbb{T} ; \operatorname{int} \operatorname{dom} h)$ (where the interior is with respect to the supremuт norm).

Proof. Let us verify the assumptions of Theorem 3. By Proposition 1, the mapping $t \mapsto$ $\mu$-liminfdom $h_{t}$ is inner semicontinuous solid convex-valued. Let $\hat{y} \in C(\mathbb{T} ; \operatorname{int} \mu$-liminf $\operatorname{dom} h)$; such $\hat{y}$ exists by [10, Theorem 3.1"']. By [14, Lemma 2], there is an $r>0$ with $\hat{y}_{t}+x \in \operatorname{int} \mu$-liminfdom $h_{t}$ for all $t$ whenever $|x|<r$; see [14, p. 460]. By Proposition $11 \mathbb{B}\left(\hat{y}_{t}, r\right) \subset \operatorname{dom} h_{t} \mu$-a.e. so that $\hat{y} \in \operatorname{dom} I_{h}$. Moreover, by [14, Theorem 2], there is a $w \in L^{1}\left(\mathbb{T}, \mu ; \mathbb{R}^{d}\right)$ with $I_{h^{*}}(w)<\infty$. By defining $\bar{\theta} \in M^{b}$ as $d \bar{\theta} / d \mu=w$ and $\bar{\theta}^{s}=0$, we have that $J_{h^{*}}(\bar{\theta})<\infty$. Therefore, Theorem 3 is applicable, and consequently $\operatorname{dom} h$ is outer $\mu$-regular if and only if $I_{h}$ and $J_{h}^{*}$ are conjugates of each other.

Assume that dom $h$ is outer $\mu$-regular. As above, we get that int $\operatorname{dom} I_{h} \supset C(\mathbb{T}$; int dom $h)$. Assume that $\bar{y} \in \operatorname{int} \operatorname{dom} I_{h}$. There is an $r>0$ such that $\bar{y}_{t}+x \in \operatorname{dom} h_{t} \mu$-a.e. whenever $|x|<r$. By Theorem 11 we have $\bar{y}_{t}+x \in \operatorname{dom} h_{t}$ for all $t$ whenever $|x|<r$. Thus $\bar{y}_{t} \in \operatorname{int} \operatorname{dom} h_{t}$ for all $t$ and int $\operatorname{dom} I_{h} \subseteq C(\mathbb{T} ; \operatorname{int} \operatorname{dom} h)$.

\subsection{Integral functionals on left continuous functions of bounded variation}

Let $\tau$ and $\tau_{l}$ be, respectively, the standard topology and the left half-open topology on $\mathbb{R}$. Here $\tau_{l}$ is generated by the basis $\{(s, t] \mid s<t\}$. The space $\left(\mathbb{R}, \tau_{l}\right)$ is a perfectly normal Lindelöf $T_{1}$-space; in particular it is strongly Lindelöf and $C\left(\left(\mathbb{R}, \tau_{l}\right) ; \mathbb{R}^{d}\right)$ is the space of left continuous functions (see [17, p. 75] [7, p. 194]). We say that a set-valued mapping $\Gamma:[0, T] \rightrightarrows \mathbb{R}^{d}$ is left-inner semicontinuous, if it is inner semicontinuous with respect to $\tau_{l}$. Similarly we say that $\Gamma$ is left-outer $\mu$-regular if $\Gamma$ is outer $\mu$-regular with respect to $\tau_{l}$.

Let $\left(\Gamma^{\alpha}\right)_{\alpha \in \mathcal{G}}$ be a family of $\mathbb{R}^{d}$-valued set-valued mappings. A closed-valued mapping $\Gamma$ is called $\mu$-essential supremum of $\left(\Gamma^{\alpha}\right)_{\alpha \in \mathscr{J}}$ if $\Gamma_{t}^{\alpha} \subseteq \Gamma_{t} \mu$-a.e. for all $\alpha \in$ $\mathscr{J}$, and if $\tilde{\Gamma}$ is another closed-valued mapping satisfying $\Gamma_{t}^{\alpha} \subseteq \tilde{\Gamma}_{t} \mu$-a.e. for all $\alpha \in \mathscr{J}$, then $\Gamma_{t} \subseteq \tilde{\Gamma}_{t} \mu$-a.e. When $\mu$ is $\sigma$-finite, $\mu$-essential supremum exists (see, e.g., [19, Theorem 1.3]), it is defined almost everywhere and it is denoted by $\mu$ - $\operatorname{esssup}_{\alpha \in \mathscr{J}} \Gamma^{\alpha}$.

We denote the space of left continuous $\mathbb{R}^{d}$-valued functions of bounded variation on $\mathbb{R}$ by $B V$. 
Theorem 4. Assume that $\mu$ is a $\sigma$-finite Radon measure on $([0, T], \tau), h$ is a convex normal integrand on $[0, T] \times \mathbb{R}^{d}$ with $\operatorname{dom} h$ left-inner semicontinuous, int $\operatorname{dom} h_{t} \neq \emptyset$ for all $t$, and that $I_{h}$ is finite on the nonempty set

$$
\left\{x \in B V \mid \exists r>0: \mathbb{B}\left(x_{t}, r\right) \subset \operatorname{dom} h_{t} \mu \text {-a.e. } t \in[0, T]\right\} .
$$

Then $\operatorname{dom} h$ is left-outer $\mu$-regular if and only if, for every $\theta \in M_{b}\left([0, T], \tau ; \mathbb{R}^{d}\right)$,

$$
\sup _{x \in B V}\left\{\int_{[0, T]} x_{t} d \theta_{t}-\int_{[0, T]} h_{t}\left(x_{t}\right) d \mu_{t}\right\}=J_{h^{*}}(\theta) .
$$

Proof. By [14, Theorem 2], there is a $w \in L^{1}\left(\mathbb{T}, \mu ; \mathbb{R}^{d}\right)$ with $I_{h^{*}}(w)<\infty$. Thus, as in (6), $I_{h}(x)>-\infty$ for all $x \in B V$. Let $\bar{B} \in \mathscr{B}(T)$ be such that $\mu\left(\bar{B}^{C}\right)=\left|\theta^{s}\right|(\bar{B})=0$. We define

$$
\bar{h}_{t}(x)= \begin{cases}-x \cdot\left(\frac{d \theta}{d \mu}\right)_{t}+h_{t}(x) & \text { if } t \in \bar{B} \\ -x \cdot\left(\frac{d \theta^{s}}{d \mid \theta^{s}}\right)_{t} & \text { if } t \in \bar{B}^{C}\end{cases}
$$

and $\mu^{\theta}=\mu+\left|\theta^{s}\right|$ so that

$$
\int_{[0, T]} x_{t} d \theta_{t}-\int_{[0, T]} h_{t}\left(x_{t}\right) d \mu_{t}=\int_{[0, T]}-\bar{h}_{t}\left(x_{t}\right) d \mu_{t}^{\theta} .
$$

The space $B V$ is PCU-stable in the sense that, for any $\left(x^{i}\right)_{i=0}^{n} \subset B V$ and for any continuous partition of unity $\left(\alpha^{i}\right)_{i=0}^{n}$ such that $\alpha^{0} \in C^{\infty}(\mathbb{R} ;[0,1]), \alpha^{i} \in C_{c}^{\infty}(\mathbb{R} ;[0,1])$, we have $\sum_{i=0}^{n} \alpha^{i} x^{i} \in B V$. By extending $\bar{h}$ to $\mathbb{R} \times \mathbb{R}^{d}$ by zero we get, by [6, Theorem 1], that

$$
\inf _{x \in B V} \int_{[0, T]} \bar{h}_{t}\left(x_{t}\right) d \mu_{t}^{\theta}=\int_{[0, T]} \inf _{x \in \Gamma_{t}} \bar{h}_{t}(x) d \mu_{t}^{\theta},
$$

where $\Gamma=\mu^{\theta}$ - $\operatorname{esssup}_{x \in B V \cap \operatorname{dom} I_{h}} x$.

Let us prove that $\Gamma_{t}=\mu$-liminfdom $h_{t} \mu^{\theta}$-a.e., where $\mu$-liminf is with respect to $\tau_{l}$. By Proposition 1 and Theorem $11 x_{t} \in \mu$-liminfdom $h_{t}$ for all $t$ whenever $x \in B V \cap$ $\operatorname{dom} I_{h}$. Therefore it suffices to show that $\mu$-liminfdom $h$ is smaller than the essential supremum.

Let $\bar{x} \in B V$ and $\bar{r}>0$ be such that $\mathbb{B}\left(\bar{x}_{t}, \bar{r}\right) \in \operatorname{dom} h_{t} \mu$-a.e. By Proposition 1 , $\mu$-liminfdom $h$ is left-inner semicontinuous solid convex-valued. Let $t \in \mathbb{R}$ and $\hat{x} \in$ $\operatorname{int} \mu$-liminfdom $h_{t}$. It follows from [16, Theorem 5.9] that there is an interval $(s, t]$ and $\hat{r}>0$ such that $\mathbb{B}(\hat{x}, \hat{r}) \subset \operatorname{int} \mu$-liminfdom $h_{t^{\prime}}$ for all $t^{\prime} \in(s, t]$. By Proposition 1 , $\mathbb{B}(\hat{x}, \hat{r}) \subset \operatorname{dom} h_{t^{\prime}} \mu$-a.e. on $(s, t]$ so that there is an $r<\hat{r}$ such that $x_{t}=\bar{x}_{t} 1_{[0, s]}+\hat{x} 1_{(s, t]}+$ $\bar{x}_{t} 1_{(t, T]}$ satisfies $\mathbb{B}\left(x_{t}, r\right) \subseteq \operatorname{dom} h_{t} \mu$-a.e. Therefore, for any $t$ and $\hat{x} \in \operatorname{int} \mu$-liminfdom $h_{t}$ there is $x \in B V \cap \operatorname{dom} I_{h}$ with $x_{t}=\hat{x}$. This implies that, for any $t$ and $x^{\prime} \in \mathbb{R}^{d}$,

$$
d\left(x^{\prime}, \mu \text {-liminfdom } h_{t}\right)=\inf \left\{\left|x^{\prime}-x_{t}\right| \mid x \in B V \cap \operatorname{dom} I_{h}\right\} .
$$

Let $D$ be a countable dense set in $\mathbb{R}^{d}$. As in the proof of [19, Theorem 1.3], there is a sequence $\left(x^{v}\right)_{v=1}^{\infty} \subset B V \cap \operatorname{dom} I_{h}$ such that $\left(\mu^{\theta} \text { - } \operatorname{esssup}_{x \in B V \cap \operatorname{dom} I_{h}} x\right)_{t}=\operatorname{cl} \bigcup_{v=1}^{\infty} x_{t}^{v}$ $\mu^{\theta}$-a.e. and, for every $x^{\prime} \in D$,

$$
\inf \left\{\left|x^{\prime}-x_{t}\right| \mid x \in B V \cap \operatorname{dom} I_{h}\right\} \geq \inf \left\{\left|x^{\prime}-x_{t}^{v}\right| \mid v \geq 1\right\} \quad \mu^{\theta} \text {-a.e. }
$$


This together with (9) implies that $\mu$-liminfdom $h_{t} \subseteq\left(\mu^{\theta} \text { - } \operatorname{esssup}_{x \in B V \cap \operatorname{dom} I_{h}} x\right)_{t} \mu^{\theta}$-a.e.

Since $\mu$-liminf $\operatorname{dom} h_{t}=\Gamma_{t} \mu^{\theta}$-a.e., and $I_{\bar{h}}^{\theta}$ is proper on $B V$, we get, by $(\mathbb{8})$, that

$$
\begin{aligned}
\sup _{x \in B V}\left\{\int_{\mathbb{R}} x_{t} d \theta_{t}-\int_{\mathbb{R}} h_{t}\left(x_{t}\right) d \mu_{t}\right\} & =-\inf _{x \in B V} \int_{\mathbb{T}} \bar{h}_{t}\left(x_{t}\right) d \mu_{t}^{\theta} \\
& =-\int_{\mathbb{T} x \in \mu \text {-liminfdom } h_{t}} \bar{h}_{t}(x) d \mu_{t}^{\theta} .
\end{aligned}
$$

Assuming that dom $h$ is left-outer $\mu$-regular, similarly to (7), the above expression implies the claim. Assume now that dom $h$ is not left-outer $\mu$-regular. By (3) and the fact that $\left(h_{t}^{*}\right)^{\infty}(v)=\sup \left\{v \cdot x \mid x \in \operatorname{dom} h_{t}\right\}$ (see [13, Theorem 13.3]), there is $\bar{t}$ and $\bar{v} \in \mathbb{R}^{d}$ such that $\sup \left\{\bar{v} \cdot x \mid x \in \mu\right.$-liminfdom $\left.h_{\bar{t}}\right\}>\left(h_{\bar{t}}^{*}\right)^{\infty}(\bar{v})$. Here $\mu$ cannot have an atom at $\bar{t}$, otherwise $\{\bar{t}\} \in \mathscr{H}_{\bar{t}}^{\mu \#}$ and $\mu$-liminfdom $h_{\bar{t}} \subseteq \operatorname{cldom} h_{\bar{t}}$. Let $\theta=\bar{\theta}+\bar{v} \delta_{\bar{t}}$, where $\delta_{\bar{t}}$ denotes a Dirac measure at $\bar{t}$ and $\bar{\theta}$ is absolutely continuous with respect to $\mu$ with $d \bar{\theta} / d \mu=w$. By Proposition 1 cldom $h_{t}=\mu$-liminfdom $h_{t} \mu$-a.e. so that

$$
\begin{aligned}
\sup _{x \in B V}\left\{\int_{\mathbb{R}} x_{t} d \theta_{t}-\int_{\mathbb{R}} h_{t}\left(x_{t}\right) d \mu_{t}\right\} & =-\int_{\mathbb{T} x \in \mu \text {-liminfdom } h_{t}} \bar{h}_{t}(y) d \mu_{t}^{\theta} \\
& =\int_{\mathbb{T}} h_{t}^{*}\left(\left(\frac{d \theta}{d \mu}\right)_{t}\right) d \mu_{t}+\sup _{x \in \mu \text {-liminfdom } h_{\bar{t}}} \bar{v} \cdot x \\
& >J_{h^{*}}(\theta) .
\end{aligned}
$$

\section{References}

[1] Ambrosio, L., Buttazzo, G.: Weak lower semicontinuous envelope of functionals defined on a space of measures. Ann. Mat. Pura Appl. (4) 150, 311-339 (1988). DOI 10.1007/BF01761473. URL http://dx.doi.org/10.1007/BF01761473

[2] Ambrosio, L., Fusco, N., Pallara, D.: Functions of bounded variation and free discontinuity problems. Oxford Mathematical Monographs. The Clarendon Press Oxford University Press, New York (2000)

[3] Attouch, H., Buttazzo, G., Michaille, G.: Variational Analysis in Sobolev and BV Spaces: Applications to PDEs and Optimization, MPS/SIAM Series on Optimization, vol. 6. Society for Industrial and Applied Mathematics (SIAM), Philadelphia, PA (2005)

[4] Bagh, A., Wets, R.: Convergence of set-valued mappings: equi-outer semicontinuity. Set-Valued Anal. 4(4), 333-360 (1996). DOI 10.1007/BF00436110. URL http://dx.doi.org/10.1007/BF00436110

[5] Bogachev, V.I.: Measure theory. Vol. II. Springer-Verlag, Berlin (2007). DOI 10.1007/978-3-540-34514-5. URL http://dx.doi.org/10.1007/978-3-540-34514-5 
[6] Bouchitté, G., Valadier, M.: Integral representation of convex functionals on a space of measures. J. Funct. Anal. 80(2), 398-420 (1988). $\quad$ DOI 10.1016/0022-1236(88)90009-2. URL http://dx.doi.org/10.1016/0022-1236(88)90009-2

[7] Engelking, R.: General topology, Sigma Series in Pure Mathematics, vol. 6, second edn. Heldermann Verlag, Berlin (1989). Translated from the Polish by the author

[8] Gardner, R.D., Pfeffer, V.F.: Interrelation between the regularity and $\sigma$-finiteness of Radon measures. Uspekhi Mat. Nauk 35(3(213)), 31-36 (1980). International Topology Conference (Moscow State Univ., Moscow, 1979), Translated from the English by A. V. Arhangel'ski

[9] Kabanov, Y.M., Safarian, M.: Markets with transaction costs. Springer Finance. Springer-Verlag, Berlin (2009). DOI 10.1007/978-3-540-68121-2. URL http://dx.doi.org/10.1007/978-3-540-68121-2. Mathematical theory

[10] Michael, E.: Continuous selections. I. Ann. of Math. (2) 63, 361-382 (1956)

[11] Munkres, J.: Topology, 2 edn. Prentice Hall (2000)

[12] Pennanen, T., Perkkiö, A.P.: Duality for convex problems of Bolza over functions of bounded variation. manuscript

[13] Rockafellar, R.T.: Convex analysis. Princeton Mathematical Series, No. 28. Princeton University Press, Princeton, N.J. (1970)

[14] Rockafellar, R.T.: Integrals which are convex functionals. II. Pacific J. Math. 39, 439-469 (1971)

[15] Rockafellar, R.T.: Dual problems of Lagrange for arcs of bounded variation. In: Calculus of variations and control theory (Proc. Sympos., Math. Res. Center, Univ. Wisconsin, Madison, Wis., 1975; dedicated to Laurence Chisholm Young on the occasion of his 70th birthday), pp. 155-192. Publ. Math. Res. Center Univ. Wisconsin, No. 36. Academic Press, New York (1976)

[16] Rockafellar, R.T., Wets, R.J.B.: Variational analysis, Grundlehren der Mathematischen Wissenschaften [Fundamental Principles of Mathematical Sciences], vol. 317. Springer-Verlag, Berlin (1998)

[17] Steen, L.A., Seebach, J.J.A.: Counterexamples in topology. Dover Publications Inc., Mineola, NY (1995). Reprint of the second (1978) edition

[18] Valadier, M.: Fonctions et opérateurs sur les mesures. Formules de dualité. In: Séminaire d'analyse convexe, Vol. 16, pp. Exp. No. 3, 31. Univ. Sci. Tech. Languedoc, Montpellier (1986)

[19] Wang, R.: Essential (convex) closure of a family of random sets and its applications. J. Math. Anal. Appl. 262(2), 667-687 (2001). DOI 10.1006/jmaa.2001. 7596. URL http://dx.doi.org/10.1006/jmaa.2001.7596 